\title{
GUIDELINES FOR VACCINATION OF DOGS IN SRI LANKA
}

\author{
Indira Silva
}

Veterinary Teaching Hospital, University of Peradeniya, Sri Lanka

\begin{abstract}
A vaccination is active immunization done to induce an immune response in an individual to achieve many goals, such as, protection from infectious agents (microorganisms, parasites) and immunotherapy for allergies, tumours, etc. Vaccinations are immunostimulants used in Immunotherapy to stimulate the immune system to protect an individual from disease. A vaccine imitates a pathogen, thus inducing the immune response in a manner similar to a natural infection, without exposing the individual to the virulent agent.
\end{abstract}

\section{Types of Vaccines}

Different types of vaccines are available based on the manufacturing technique, namely, inactivated (killed) vaccines, modified live attenuated vaccines (MLV), toxoids, recombinant vectored vaccines, subunit vaccines, Anti-idiotype vaccine and DNA vaccines. These vaccines stimulate the immune system to produce either a humoral response $(\mathrm{HI})$, or a cell-mediated response (CMI), or both.

Currently, the companion animal vaccines are simply considered as either 'infectious' or 'noninfectious' in nature. Most infectious vaccines used in dogs are attenuated or modified live virus (MLV) vaccines in which the organisms are intact and viable and can induce immunity by inducing a low-level infection and replication within the body, without producing significant tissue pathology or clinical signs (Day, 2016). Some recombinant vectored vaccines are also considered 'infectious'. The MLV vaccines induce an effective immunity when administered parenterally at recommended anatomical sites, and are more likely to induce robust CMI and HI (Day, 2016). Some MLV vaccines are administered directly to mucosal sites, such as nasal and oral vaccines, which effectively induce protective mucosal immunity. The non-infectious vaccines, which include killed/inactivated vaccines, subunit vaccines or DNA vaccines, contain an inactivated but antigenically intact virus or organism, a natural or synthetic antigen from the pathogen, or DNA that can encode such an antigen. They generally require an adjuvant to increase their potency and also require multiple doses to induce protection. The noninfectious vaccines are administered parenterally, less likely to induce both HI and CMI, and have a shorter Duration of Immunity (DOI) compared to infectious vaccines.

It is important to reduce the "vaccine load" on individual animals in order to minimize the potential adverse reactions to vaccine products, to reduce the time and financial burden on clients and veterinarians (Day, 2016). The author has observed evidence of Immune-mediated hemolysis, such as autoagglutination of RBC and ghost cells in clinically healthy dogs that have been vaccinated annually with MLV vaccines, according to past vaccination protocols. Since such an immune mediated hemolysis could be attributed to multiple vaccinations with MLV vaccines (CSU 2013), vaccination guidelines should be based on rational analysis of vaccine requirements for each pet, categorized as core vaccines, non-core vaccines and not recommended vaccines (Day, 2016). All dogs should receive 'Core Vaccines' to protect against infectious diseases of global significance. The core vaccines for dogs in Sri Lanka are: canine distemper virus (CDV), canine adenovirus (CAV types 1 and 2), canine parvovirus type $2(\mathrm{CPV}-2)$ and its variants, and Rabies, which was included in this category due to the endemic nature of the disease in Sri Lanka.

The use of 'Non-Core Vaccines' is dictated by geographical location, the lifestyle and exposure risks of the individual animal, and judging the risk of being unvaccinated and susceptible versus the benefit of being protected against the infection. The 'Not recommended vaccines' are those with insufficient scientific justification for their use.

The vaccine data sheets submitted by companies as part of the registration process, provides details of the quality, safety, efficacy and the minimum Duration of Immuity (DOI) of the vaccine product (Day 2016). The DOI in the data sheet is based on experimental evidence and need not reflect the true DOI of a vaccine. Most core vaccines, until relatively recently, had a 1-year minimum DOI requiring annual revaccination, but are now licensed with a minimum DOI of 3-4 years (Day 2016). 


\section{Herd Immunity}

Vaccination is one of the most debated topics in veterinary medicine, because while wanting to protect companion animals from deadly infectious diseases, we are also concerned with the problems created by over-vaccination. Every effort should be taken to achieve a large number of individually vaccinated animals in a population (at least $70 \%$ ) to provide a long DOI of many years capable of protecting all susceptible animals in a given region, rather than increasing annual vaccinations of a smaller group (Day 2016). It has been shown that a dog receiving a core MLV vaccine every 3 years is equally well protected as one receiving the same vaccine annually, and there is no advantage in giving repeated vaccinations to an individual dog to induce better immunity (Bohm et al., 2004; Mouzin et al., 2004, Mitchell et al., 2012).

\section{Maternally Derived Antibodies}

Every effort must be taken to emphasize immunizing animals, especially puppies, rather than arbitrarily vaccinating large numbers of animals. Most puppies are protected by Maternally Derived Antibodies (MDA) in the first weeks of life through colostrum causing passive immunization. The MDAs can block vaccinal antigens, thereby requiring administration of a series of vaccines to young animals. When passive immunity wane off by $8-12$ weeks of age (MDA T1/2 of 12 days), active immunization is required to protect the young. A puppy with a high MDA titre can block vaccinal antigens and will be incapable of responding to vaccination until $\geq 12$ weeks of age. On the other hand, a puppy with a low MDA is vulnerable to infectious diseases, and is therefore capable of responding to vaccination at an earlier age. Some MLV vaccines with a high antigenic titre and some genetically modified vaccines are able to generate immunity in the presence of MDA earlier than noninfectious vaccines.

\section{Single and multi-component vaccines}

Even though a number of single and multicomponent vaccines are currently available with a benefit/risk assessment of the product, the veterinarians should be comfortable in making a clinical benefit/risk judgment based on the individual animal's age, health status, home \& travel environment, and lifestyle (FitzGerald 2000). Guidelines are necessary for this purpose, for cross referencing with local requirements and recommendations. A multivalent vaccine must prove that each component of the vaccine can induce protective immunity in challenge studies. While multicomponent core MLV vaccines (e.g. for CDV,
CAV-2 and CPV-2) are ideal for delivery of core vaccinations, individual vaccines are better for noncore antigens (e.g. Leptospira) which may be given after a risk:benefit analysis.

A dog presented with no previous history of vaccination should be able to respond to antigens in multiple vaccines delivered simultaneously in a single visit. However, different vaccines should never be mixed in the same syringe unless specifically indicated by the datasheet (eg: DH\&L). It is good practice to deliver the multiple vaccines to different anatomical sites so that different lymph nodes are involved in generating the adaptive immune response, although no studies have formally proven this (Day 2016).

\section{Revaccinations}

Annual revaccinations are recommended for noncore vaccines, but are not recommended for core vaccines. An adult dog that had received a complete course of core vaccinations as a puppy, including boosters, but have not been vaccinated regularly as an adult, or an adopted adult dog ( $>16$ wk of age) of unknown vaccination history, requires only a single dose of MLV core vaccine to get protective immunity, as solid immunological memory can be achieved with MLV vaccines (Mouzin et al., 2004; Mitchell et al., 2012). After the 52 week booster, those dogs should receive revaccinations at intervals $\geq 3$ years. Triennial revaccination does not apply to killed core vaccines, for non-core vaccines, and vaccines containing bacterial antigens.

Serological testing is an alternative to routine core revaccinations. Even though they are relatively expensive, rapid and simple in-practice kits to detect protective antibody level in individual animals are useful to monitor immunity to vaccines. The Virus Neutralization and Haemagglutination Inhibition tests are the 'gold standards' recommended for serological testing for vaccinal titres. Pups should be tested at least 4 weeks after the final vaccination to ensure maternally derived antibodies (MDA) are no longer present and 'slow responders' have seroconverted.

A 'booster' vaccine is an integral part of core vaccination given to ensure a protective immune response in any dog that may have failed to respond to a core vaccine. The priming and boosting can be done in two ways. In homologous prime-boost approach, the same formulation is used to prime and boost, while different formulations are used sequentially in more than one administration in the Heterologous prime-boost approach. The heterologous approach can stimulate both HI \& CMI and thereby is more effective, especially against intracellular pathogens and highly complex infectious agents. For instance, a DNA vaccine can be used as a 
priming vehicle and an attenuated virus as a booster vaccine. The "window of susceptibility", which is the period during which a pup can be infected by field virus but vaccines cannot immunize, is comparatively less ( $\leq 2$ wks) for highly effective MLV vaccines which has a high titre and low passage. A single dose of a MLV vaccine can prime, immunize, and boost, in the absence of MDA. Nevertheless 2 doses are recommended, in young animals with MDA, to make sure a vaccine is given when MDA has waned and cannot block vaccinal antigens. If the first dose gets blocked by MDA, the young animal will not be immunized from the second dose, thereby requiring a $3^{\text {rd }}$ dose to immunize and boost. As per recommendations of the global veterinary community, the last dose should be given at $\geq 16$ weeks of age, regardless of the number of doses given earlier. Some dog owners may wish to practice 'early finish' vaccination schedules, with the last dose given at 10 weeks, to allow early socialization of pups. The immunological validity of this approach is questioned by the WSAVA VGG, as no combination core product will immunize an acceptable percentage of puppies (particularly not against CPV-2) when the last dose is given at 10 weeks of age. Pups that have not completed a full puppy vaccination series should be carefully exposed to environments outside the home and only permit contact with healthy and fully vaccinated dogs.

\section{The need for guidelines}

Vaccination guidelines have been developed in global context, since the late 1970s. The $67^{\text {th }}$ Executive Committee of the SLVA 2014/2015, organized a seminar titled "Together Against Rabies for a Rabies Free Sri Lanka 2020" on $26^{\text {th }}$ September 2014, co-sponsored by the State Department of Animal Production and Health (DAPH) and the World Organization for Animal Health (OIE - Office International des Épizooties). This activity was preceded by two meetings at Peradeniya and Colombo organized by the "Veterinary Teaching Hospital Outreach Activity" program to design a vaccination schedule for dogs in Sri Lanka with the participation of small animal practitioners, DAPH, Ministry of Health, SLVA members and wellwishers. The schedule agreed upon at those meetings was widely circulated among the government and private veterinary practitioners.

It is important to continually reevaluate vaccination guidelines in order to get a successful response from vaccinations, as seen in developed countries where some major infectious diseases of dogs are considered as most uncommon in the pet population (Day 2016). It has been estimated that only $30-50 \%$ of pets in developed countries get vaccinated, and the number is less in developing countries. However, even in developed countries, geographical pockets of infection and sporadic outbreaks can occur. Furthermore, the situation with free-roaming dogs and shelter populations is distinctly different from that in owned pets, as their chances of exposure to wildlife are high. A majority of newly emergent human diseases is proposed to have derived from wild or domestic animals (Gibbs 2014).

Subsequently, in January 2016, the Journal of Small Animal Practice published "Guidelines for the vaccination of dogs and cats" compiled by the Vaccination Guidelines Group (VGG) of the World Small Animal Veterinary Association (WSAVA) with new updates very applicable and useful for small animal practitioners in Sri Lanka (Day 2016). The WSAVA guidelines were developed by a panel of experts based on their own 'Evidence-Based Veterinary Medicine' (EBVM) classification consisting of 4 categories. Thereafter, in May 2016 a group of senior academics of the Veterinary Teaching Hospital (VTH), the Director General and a delegation of scientists/administrators from the DAPH met and discussed the proposed new vaccination schedule for household dogs in Sri Lanka, taking in to consideration, the optimum time a dog is capable of immunologically responding to vaccinations, and the financial and social constraints faced by pet owners in Sri Lanka. Most of the facts relevant to Sri Lankan veterinarians given in the WSAVA 2016 publication are included in this publication, to the best of my capability.

When recommendations in guidelines differ from those in the product label, such as annual ARV vaccinations in Sri Lanka using products with a minimum 3 year DOI, the practitioner may obtain informed consent of the client in order to use the vaccine in accordance with the guidelines recommended.

\section{Anti-Rabies Vacine (ARV)}

The OIE states that Rabies control is a national responsibility, and the dog (principal reservoir) should be considered a main target for rabies elimination. The Ministry of Health has reported 34 cases of human Rabies in 2015 with the maximum number (10) from Kurunegala ( $\mathrm{MOH}$ Weekly Epidemiological Report), while a total number of 37 cases had been reported in 2012, with 1-5 cases distributed in many districts. Rabies is listed as a contagious disease in dogs \& cats in the Animal Diseases Act No. 59 of 1992 and the Gazette Extraordinary of the Democratic Socialist Republic of Sri Lanka dated $17^{\text {th }}$ June 2014 declared Rabies as one of 13 diseases under the category of multiple diseases species in the Animal Diseases Act. The Act 
states the immunization of animals should be done under the authority of the Director General of the DAPH who has powers to ensure the efficient and safe use of veterinary drugs and biological products on animals.

All animal rabies vaccines are not similar in efficacy, safety, stability, convenience, and supply reliability. Killed rabies vaccines produced on continuous cell lines in large scale offer the best protection and economic value. Most rabies vaccines are prepared from Pasteur's original 1882 strain and its derivative strains (Pasteur Virus, Challenge Virus Standard [CVS], Pitman-Moore), and strains isolated in the $20^{\text {th }}$ century (Flury, low egg passage [LEP], high egg passage [HEP], Street-Alabama-Dufferin [SAD], Vnukovo) (Geue et al., 2008; Tao et al., 2010; Wu et al., 2011). Rabies vaccines produced in compliance with OIE requirements protect against all variants of rabies virus including other phylogroup 1 lyssaviruses (Brookes et al., 2005). The DOI should be determined in vaccinated animals of the target species, and vaccines should confer protective immunity for at least 1 year (OIE 2013).

The Centers for Disease Control and Prevention (CDC) surveys in the United States have shown small mammals such as squirrels, rats, mice, hamsters, guinea pigs, gerbils, chipmunks, rabbits, and hares are almost never found to be infected with rabies and have not been known to cause rabies among humans. Bites by these animals are usually not considered a risk of rabies unless the animal was sick or behaving in any unusual manner and rabies is widespread in the area. However from 1985 through 1994, groundhog (Marmota monax) also known as a woodchuck, or whistle pig, had accounted for $86 \%$ of the 368 cases of rabies among rodents reported to CDC. In all cases involving rodents, the state or local health department is consulted before a decision is made to initiate post exposure prophylaxis (PEP).

If a properly vaccinated dog, after receiving the initial puppy rabies vaccines, is bitten by a freeroaming dog, it should be protected against rabies (WSAVA VGG). In some countries, PEP is given for the benefit of the puppy, and more importantly for the benefit of the human family. Repeated PEP is not justified if the same pup is bitten again some weeks later, because by that time the puppy would have received multiple vaccinations and further injections will provide no added benefit.

The canine and feline rabies is controlled mostly by inactivated vaccines which has a DOI of 3 years. A recombinant canarypox vectored vaccine is used on cats in USA and Europe because it is not associated with inflammation at the injection site. All initial ARV vaccinations should be given not earlier than 12 weeks of age (other than stray or shelter dogs) and must be followed by a second vaccination one year later. After the second vaccination, it is advisable to give annual revaccinations even if the product has a DOI label of 3 years, as Rabies is endemic in Sri Lanka. In non-endemic countries 3 year revaccinations are done after the $2^{\text {nd }}$ dose given one year after the initial vaccine. Antibody titres of $\geq 0.5 \mathrm{IU} / \mathrm{ml}$ in an actively immunized $\operatorname{dog}>16$ weeks of age is correlated with protection. Protective titres are reached 4 weeks after vaccination and antibody titres of $\geq 0.5 \mathrm{IU} / \mathrm{ml}$ is considered a legal requirement for pet travel.

\section{Canine Parvovirus Vaccine (CPV-2)}

A majority of exposed animals get life-long DOI after natural infection/disease. The 3 variants of CPV-2 (CPV-2a, CPV-2b and CPV-2c) are antigenically related, and current $\mathrm{CPV}$ vaccines containing either $\mathrm{CPV}-2$ or $\mathrm{CPV}-2 \mathrm{~b}$ provide protective immunity against all variants (Spibey et al., 2008; Decaro \& Buonavoglia 2012; Wilson et al., 2013). The MLV vaccines are the most common products, with a 9 year DOI in the absence of MDA. Re-vaccinations are recommended every 3 years after the vaccination at 26 or 52 months of age. Puppies less than 4-6 weeks of age, pregnant bitches and wildlife should not be vaccinated with the MLV vaccine. An outbreak of CPV-2c infection had been reported in vaccinated adult dogs aged between 6 months and 2.5 years in a breeding kennel in Italy, that had been repeatedly administered CPV-2 (old type) vaccine at 6 weeks, 8 weeks and 90 days of age with annual vaccinations of adults (Decaro et al., 2008). Inactivated vaccines are less effective, and the DOI is only for $\geq 3$ years. The presence of serum antibodies, regardless of the titre, in an actively immunized dog of $>20$ weeks age is correlated with protection. The CPV-2 vaccines with higher viral titres $\left(10^{5.5}\right.$ CCID50) and/or with more immunogenic isolates will immunize a few weeks earlier than other standard CPV-2 vaccines, and such vaccines are currently available in Sri Lanka.

\section{Canine Adenovirus Vaccine (CAV-2)}

The MLV CAV-2 is the only vaccine recommended for the prevention of Infectious Canine Hepatitis (ICH) caused by CAV-1 and also reduces signs of respiratory disease with CAV-2. No adverse reactions are observed unlike with $\mathrm{CAV}-1$ vaccines, which can cause allergic uveitis known as 'blue eye'. The MLV vaccines protect as early as 5 days post vaccination, in the absence of MDA. The DOI after naturally-acquired ICH is life-long, while DOI with MLV vaccination persist for $\geq 9$ years. Revaccination after the booster at 26 or 52 weeks should be done every 3 years. Inactivated (Killed) Vaccines are less 
effective and not recommended when MLV products are available. Intranasal CAV-2 vaccine is intended as an aid in the prevention of upper respiratory disease caused by CAV-2 and is not intended to protect against ICH infection. Puppies < 4-6 weeks of age and wildlife should not be vaccinated with the MLV vaccine. The presence of serum antibodies, regardless of the titre, in an actively immunized dog of $>20$ weeks age is correlated with protection.

\section{Distemper}

The products of MLV vaccine contain the CDV strains Rockborn, Snyder Hill, Onderstepoort, Lederle, etc., which provide protective immunity against any pathotype. Puppies $<6$ weeks of age should not be vaccinated with MLV products, unless the product has a specific license. Revaccination after the booster at 26 or 52 weeks should be done every 3 years. The MLV vaccine should not be used on wildlife and exotic species but Vectored Recombinant (rCDV) Vaccine using a canarypox virus has been used in wildlife and exotic species. Inactivated vaccines are not readily available and are not as effective as MLV vaccines. The DOI after naturally-acquired ICH is life-long in a majority of dogs, persist for $\geq 9$ years with MLV vaccination and $\geq 5$ years with rCDV. Inactivated (Killed) Vaccines are less effective and not recommended when MLV products are available.

\section{Leptospira}

Dogs can become infected and develop leptospirosis if their mucous membranes get contaminated with infected urine, urine-contaminated soil, water, food or bedding; through a bite from an infected animal; by eating infected tissues or carcasses; and intrauterine transfer from an infected mother to puppies is also possible. The Ministry of Health reports 4331 leptospirosis cases in the year 2015, highest number in Gampaha, Kalutara, Ratnapura, then Kegalle, Anuradhapura, Kurunegala, Colombo followed by Galle, Matara (MOH Weekly Epidemiological Report). In contrast a total of 2663 cases had been reported in 2012 (MOH Annual Epidemiological Bulletin 2012). Non-core Leptospira vaccines have been developed to protect against known circulating pathogenic serogroups in different geographical areas. Vaccination should be restricted to be used in geographical areas where a risk of exposure has been established for dogs whose lifestyle places them at risk. In countries where Leptospirosis in endemic, stray dogs and dogs who spend most of their time outdoors, may have higher chances of contracting leptospirosis through direct contact with contaminated water. Dogs in suburban or rural environments are at increased risk of leptospirosis presumably because of greater likelihood of contact with wildlife habitats (Ward et al., 2004). Wildlife plays a key role in the transmission of the disease in urban and periurban environments, and raccoons have been identified as a major reservoir of the pathogen (Diniz http://www.leptoinfo.com/). Indoor dogs living in urban and suburban areas, even with limited outdoor access, are also at a similar risk of infection as dogs in rural areas in contact with wildlife, livestock and stagnant water. The risk level is higher in a Labrador Retriever who may be tempted to jump into every water body than a small breed like a Pomeranian that stays indoors at all times. Veterinarians should be aware of this trend when determining a dog's risk of exposure in order to define the best prophylactic strategy. Vaccine for Leptospra is less robust and may be of shorter duration, and therefore these products require boosters annually for reliable protection (Ellis \& Krakowka 2012; Klaasen et al., 2014; Ellis 2015; Schuller et al., 2015). Even though the incidence of Leptospirosis as a result of direct transmission of the bacterium from dogs to its owners and their families is low, annual vaccination of pet dogs will protect the family against the newer and older serotypes (Brown and Prescott 2008).

Post-vaccinal antibodies generally persist only for a few months and immunological memory for protective immunity is relatively short, for about a year. There is no clear evidence that 6-monthly revaccination confers greater protection than annual revaccination in high-risk areas. Even though all Leptospira serovars $(>250)$ are not pathogenic, $>60 \%$ of the cases in dogs are caused by serovars not present in bivalent vaccines (L. grippotyphosa and $L$. pomona), which demonstrates the importance of using a vaccine against at least 4 serovars, namely, $L$. Canicola, L. icterohaemorrhagiae, L. Pomona, and L. grippotyphosa in high risk dogs (Gautam et al., 2010). In many countries, including Sri Lanka, there is insufficient knowledge of which serogroups are circulating in the canine population, and the collection of such data should be encouraged.

The CDC recommends administering leptospira vaccines separately because when given as a combination vaccine, the chance for a vaccine reaction is higher in small dogs, and particularly in the miniature dachshund. Antibody determinations to Leptospira products are of limited or no value because of the short time period these antibodies persist or the lack of correlation between serum antibody and protection (Hartman et al. 1984; Klaasen et al., 2003; Ellis \& Krakowka 2012; Martin et al., 2014). 


\section{Tetanus}

There are no licensed vaccines for dogs, as tetanus is uncommon in dogs in many parts of the world. However, nowadays tetanus is observed considerably more frequently than canine infectious hepatitis and canine distemper, and veterinarians do use equine tetanus vaccine in dogs (off-label use). Dog vaccination protocol for Sri Lanka revised and compiled by the Peradeniya Veterinary Teaching Hospital and the Department of Animal Production and Health, Getambe, based on WSAVA recommendations.

\begin{tabular}{|c|c|c|c|c|c|c|c|c|c|}
\hline \multicolumn{10}{|c|}{ Immunized Mother } \\
\hline $\begin{array}{l}\text { Vaccine } \\
\text { \& Doses } \\
\end{array}$ & $\mathbf{1}^{\text {st }}$ & $2^{\text {nd }}$ & $3^{\text {rd }}$ & $4^{\text {th }}$ & $5^{\text {th }}$ & $6^{\text {th }}$ & \multicolumn{3}{|c|}{ Boosters } \\
\hline CPV & 8 & 11 & & 14 & & 52 & \multicolumn{3}{|l|}{$3 y$} \\
\hline DHL & 8 & 11 & & & & & & & \\
\hline DH & & & & 14 & 17 & 52 & \multicolumn{3}{|l|}{$3 \mathrm{y}$} \\
\hline ARV & & & 12 & 14 & & 52 & \multicolumn{3}{|l|}{ annual } \\
\hline \multicolumn{10}{|c|}{ Non-immunized Mother } \\
\hline $\begin{array}{l}\text { Vaccine } \\
\text { \& Doses }\end{array}$ & $1^{\text {st }}$ & $2^{\text {nd }}$ & $3^{\text {rd }}$ & $4^{\text {th }}$ & $5^{\text {th }}$ & 6th & 7th & $8^{\text {th }}$ & Boosters \\
\hline ARV & $\begin{array}{l}\text { First } \\
\text { visit }\end{array}$ & & & & 12 & 14 & & 52 & annual \\
\hline CPV & & $\begin{array}{l}7 \\
(\geq 4)\end{array}$ & & 11 & & 14 & 17 & 52 & $3 y$ \\
\hline DHL & & & 8 & 11 & & 14 & 17 & 52 & $3 y$ \\
\hline
\end{tabular}

ARV post exposure vaccination

If victim properly immunized - one booster ARV

If victim unvaccinated, according to WHO Recommendations - No ARV given. Observe dog for six months for signs of Rabies. If negative vaccinate at six months

Shelter Dogs (WSAVA Recommendations)

\begin{tabular}{|l|l|l|l|}
\hline & & $\mathbf{1}^{\text {st }}$ dose & Repeat dose \\
\hline $\begin{array}{l}\text { CDV }+ \\
\text { CAV-2 }+ \\
\text { CPV-2 }+ \\
\text { Lepto }\end{array}$ & Puppies & $\begin{array}{l}\text { Prior to or immediately on } \\
\text { admission, as early as 4 weeks } \\
\text { of age }\end{array}$ & $\begin{array}{l}\text { 2 week interval until 20 weeks of age, if } \\
\text { still in the facility }\end{array}$ \\
\cline { 2 - 4 } & Adults & $\begin{array}{l}\text { Prior to or immediately on } \\
\text { admission }\end{array}$ & 2 weeks later \\
\hline ARV & & $\begin{array}{l}\text { Follow the schedule for pups from non-immunized mothers, since Rabies is } \\
\text { endemic in Sri Lanka }\end{array}$ \\
\hline
\end{tabular}

\section{REFERENCES}

Bohm, M., Thompson, H., Weir, A., Hasted, A. M., Maxwell, N. S., \& Herrtage, M. E. (2004). Serum antibody titres to canine parvovirus, adenovirus and distemper virus in dogs in the UK which had not been vaccinated for at least three years. Veterinary Record, 154(15), 457.

Brookes, S.M., Parsons G, Johnson N., Mcelhinney L.M. \& Fooks A.R. (2005). Rabies human diploid cell vaccine elicits cross-neutralising and crossprotecting immune responses against European and 
Australian bat lyssaviruses. Vaccine, 23, 41014109.

Brown, K., \& Prescott, J. (2008). Leptospirosis in the family dog: a public health perspective. Canadian Medical Association Journal, 178(4), 399-401..

Center for Companion Animal Studies, Colorado State University (CSU) (2013). Are vaccines a primary cause of hemolytic anemia or immunemediated thrombocytopenia in dogs? Veterinary Teaching Hospital,.

http://csu-

cvmbs.colostate.edu/vth/veterinarians/research/co mpanion-animals/Pages/foundations.aspx.

Day, M..J., Horzinek, M.C., Schultz, R.D., and Squires R.A. (2016). Guidelines for the vaccination of dogs and cats. Journal of Small Animal Practice, 57:E1-E45.

Decaro, N., Desario, C., Elia, G., Martella, V., Mari, V., Lavazza, A., Nardi, M. and Buonavoglia, C., (2008). Evidence for immunisation failure in vaccinated adult dogs infected with canine parvovirus type 2c. Microbiologica-Quarterly Journal of Microbiological Sciences,31(1), pp.125-130.

Diniz, P P Canine leptospirosis: Growing in effect and threat. http://www.leptoinfo.com/clinics_veterinarians/ lepto_articles/articleTwo2.html.

Ellis, J. A. (2015) How well do vaccines for Bordetella bronchiseptica work in dogs? A critical review of the literature 1977-2014. Veterinary Journal 204, 5-16

Ellis, J. A. \& Krakowka, G. S. (2012) A review of canine parainfluenza virus infection in dogs. Journal of the American Veterinary Medical Association 240, 273-284

FitzGerald, J. (2000). Canine Vaccination Schedules. The Veterinary Record. Feb 27, 2010. Pp 280-281.

Gautam, R., Wu, C.C., Guptill, L.F., Potter, A. and Moore, G. E. (2010). Detection of antibodies against Leptospira serovars via microscopic agglutination tests in dogs in the United States, 2000-2007. Journal of the American Veterinary Medical Association, 237(3), pp.293-298.

Geue, L., Schares, S., Schnick, C,, Kliemt, J., Beckert, A., Freuling, C., Conraths, F.J., Hoffmann, B., Zanoni, R., Marston, D., Mcelhinney, L., Johnson,N., Fooks, A.R., Tordo, N. \& Müller, T. (2008). Genetic characterisation ofattenuated SAD rabies virus strains used for oral vaccination of wildlife. Vaccine, 26, 3227-3235.

Gibbs, E.P.J. (2014). The evolution of one-health: a decade of progress and challenges for the future.Veterinary Record 174:85-91.

Hartman, E. G., van Houten, M., Frik, J. F. \& van der Donk, J. A. (1984) Humoral immune response of dogs after vaccination against leptospirosis measured by an IgM- and IgG-specific ELISA. Veterinary Immunology and Immunopathology 7, 245-254

Klaasen, H.L.B.M., van der Veen, M., Sutton, D. and Molkenboer, M.J.C.H. (2014). A new tetravalent canine leptospirosis vaccine provides at least 12 months immunity against infection. Veterinary immunology and immunopathology, 158(1), pp.2629.

Klaasen, H. L., Molkenboer, M. J., Vrijenhoek, M. P. \& Kaashoek, M. J. (2003) Duration of immunity in dogs vaccinated against leptospirosis with a bivalent inactivated vaccine. Veterinary Microbiology 95, 121-132

Martin, L.E.R., Wiggans, K..T., Wennogle, S.A., Curtis, K., Chandrashekar, R. and Lappin, M.R. (2014). Vaccine-Associated Leptospira Antibodies in Client-Owned Dogs. Journal of Veterinary Internal Medicine, 28(3), pp.789-792.

Mitchell, S.A., Zwijnenberg, R.J., Huang, J., Hodge, A. and Day, M.J. (2012). Duration of serological response to canine parvovirus-type 2, canine distemper virus, canine adenovirus type 1 and canine parainfluenza virus in client-owned dogs in Australia. Australian veterinary journal, 90(12), pp.468-473.

Mouzin, D.E., Lorenzen, M.J., Haworth, J.D. \& King, V.L. (2004). Duration of serologic response to five viral antigens in dogs. Journal of the American Veterinary Medical Association 224:5560.

Schuller, S., Francey, T., Hartmann, K., Hugonnard, M., Kohn, B., Nally, J. E., \& Sykes, J. (2015). European consensus statement on leptospirosis in dogs and cats. Journal of Small Animal Practice, 56(3), 159-179.

Spibey, N., Greenwood, N. M., Sutton, D., Chalmers, W. S. K., \& Tarpey, I. (2008). Canine parvovirus type 2 vaccine protects against virulent challenge with type $2 \mathrm{c}$ virus. Veterinary microbiology, 128(1), 48-55.

Tao, L. Ge J., Wang, X., Zhai, H., Hua, T., Zhao, B., Kong, D., Yang, C., Chen, H. \& Bu, Z. (2010). Molecular basis of neurovirulence of flury rabies virus vaccine strains: importance of the polymerase and the glycoprotein R333Q mutation. Journal of Virology., 84, 8926-8936.

Ward, M.P., Guptill, L.F. and Wu, C.C., (2004). Evaluation of environmental risk factors for leptospirosis in dogs: 36 cases (19972002). Journal of the American Veterinary Medical Association, 225(1), pp.72-77.

Wilson, S., Stirling, C., Borowski, S., Thomas, A., King, V., \& Salt, J. (2013). Vaccination of dogs with Duramune DAPPi+ LC protects against 
pathogenic canine parvovirus type 2c challenge. Veterinary Record: Journal of the British Veterinary Association, 172(25).

World Assembly of Delegates of the OIE in May 2011; Section C adopted in May 2013 - Chapter 2.1.13 - Rabies
Wu, X., Smith, T. G., \& Rupprecht, C. E. (2011). From brain passage to cell adaptation: the road of human rabies vaccine development. Expert review of vaccines, 10(11), 1597-1608. 